\title{
Bioinformation
}

\author{
www.bioinformation.net
}

Views \& Challenges

\section{Modeling of phosphomethyl pyrimidine kinase from Leptospira interrogans serovar lai strain 56601}

\author{
Vetrivel Umashankar ${ }^{1}$, Gurunathan Subramanian ${ }^{2}$ and Sudarsanam Dorairaj ${ }^{3 *}$ \\ ${ }^{1}$ Department of Bioinformatics, Vel's College of Science, India; ${ }^{2}$ Department of Microbiology, Vel's College of \\ Science, India; ${ }^{3}$ Department of Zoology, Loyola College, Chennai, India; \\ Sudarsanam Dorairaj - Email: zoo@loyolacollege.edu; * Corresponding author \\ received May 14, 2006; revised June 07, 2006; accepted June 9, 2006; published online June 17, 2006
}

\begin{abstract}
:
Many microorganisms, as well as plants and fungi, synthesize thiamin, but vertebrates do not produce it. Phosphomethyl pyrimidine kinase is an enzyme involved in an intermediary step of thiamin biosynthesis from purine molecules. This enzyme is absent in humans. Thus, it is a potential chemotherapeutic target for antileptospiral treatment. Structure of this enzyme from Leptospira interrogans serovar lai strain 56601 has not yet been elucidated. We used the structural template of phosphomethyl pyrimidine kinase from Thermus thermophilus HB8 for modeling the phosphomethyl pyrimidine kinase structure from Leptospira interrogans serovar lai strain 56601. The model is deposited in Protein Data Bank (PDB ID: 2G53) at RCSB. Thus, we analyse and propose the usefulness of the modeled phosphomethyl pyrimidine kinase for the design of suitable inhibitors towards the treatment of leptospirosis.
\end{abstract}

Keywords: Leptospirosis; Leptospira interrogans; modeler; homology modeling; phosphomethyl pyrimidine kinase

Background:

Leptospirosis is a zoonotic disease caused by infection with pathogenic Leptospira species. It is currently identified as a worldwide emerging infectious disease, especially in developing countries. Leptospirosis caused by Leptospira interrogans serovar lai strain 56601 is commonly prevalent in India, China, Japan, Malaysia and Singapore. These spirochaetes are transmitted to man usually either by direct or indirect contact with the urine of the infected animal. The clinical presentation of the disease is biphasic, with the acute or septicemic phase lasting about a week, followed by immune phase, characterized by antibody production and excretion of leptospires in urine. [1] Infection may be sub clinical, mild or severe, affecting various internal organs. Febrile illness of sudden onset, chills, myalgia and abdominal pain are the common symptoms in sub clinical or mild infections. Jaundice and acute renal failure are also seen in severe cases [1]. Specific antibiotic treatment using doxycycline or penicillin antibiotics has shown mixed results for mild or sub clinical infections. Treatment of severe leptospirosis (organism localized in tissues) is still unclear. [1] Availability of complete genome sequence of Leptospira interrogans serovar lai strain 56601 [2] has opened great opportunities for identifying potential drug targets by computational analysis. This can in turn improve leptospiral treatments.

Vitamin B1, in its active form thiamin pyrophosphate, is an essential cofactor for several important enzymes in carbohydrate metabolism. Many microorganisms, as well as plants and fungi, synthesize thiamin, but vertebrates do not produce it. In bacteria, it is synthesized by coupling of pyrimidine (hydroxymethyl pyrimidine; HMP) and thiazole (hydroxyethyl thiazole) moieties. Of the many enzymes involved in bacterial cell's thiamin biosynthetic pathway, phosphomethyl pyrimidine kinase plays a key role. Hence, it is a promising chemotherapeutic target. This is also known as HMP phosphokinase and it catalyses the conversion of 4-amino 2-methyl 5-phosphomethyl pyrimidine to 4-amino 2-methyl 5-hydroxymethyl pyrimidine diphosphate, an intermediary step in the conversion of purine into thiamine. [9] This enzyme is coded by thiD gene ( 822 bp in length) located on chromosome 1 of Leptospira interrogans serovar lai strain 56601. [2, 3] Here, we predict 3 Dimensional structure of Leptospira interrogans serovar lai strain 56601 phosphomethyl pyrimidine kinase employing homology modeling. The predicted 3D structure was further subjected to structural feauture analysis, aiming towards developing potent inhibitors.

\section{Methodology:}

The protein sequence (273 residues long) was obtained from the SWISS PROT database (Q8F9R1). The protein databank (PDB) contains two phosphomethyl pyrimidine kinase structures from 2 species (Salmonella tyhpimurium (PDB ID: 1JXH), Thermus thermophilus HB8 (PDB ID: 1UB0)). [4] However, structure for phosphomethyl pyrimidine kinase from Leptospira interrogans serovar lai strain 56601 was not available. Sequence analysis using BLASTP [5] against PDB sequences showed phosphomethyl pyrimidine kinase from Leptospira interrogans serovar lai strain 56601 having 38\% sequence identity (highest homology compared to other known structures) with Thermus thermophilus HB8. Therefore we used the structure of Thermus thermophilus $H B 8$ phosphomethyl pyrimidine kinase (PDB: 1UB0) as template for building homology model for Leptospira interrogans serovar lai strain 


\section{Bioinformation}

www.bioinformation.net

Views \& Challenges

56601 phosphomethyl pyrimidine kinase using global alignment in this modeling process. The MODELLER [6] by satisfaction of spatial restraints. MALIGN script of MODELLER was implemented to build

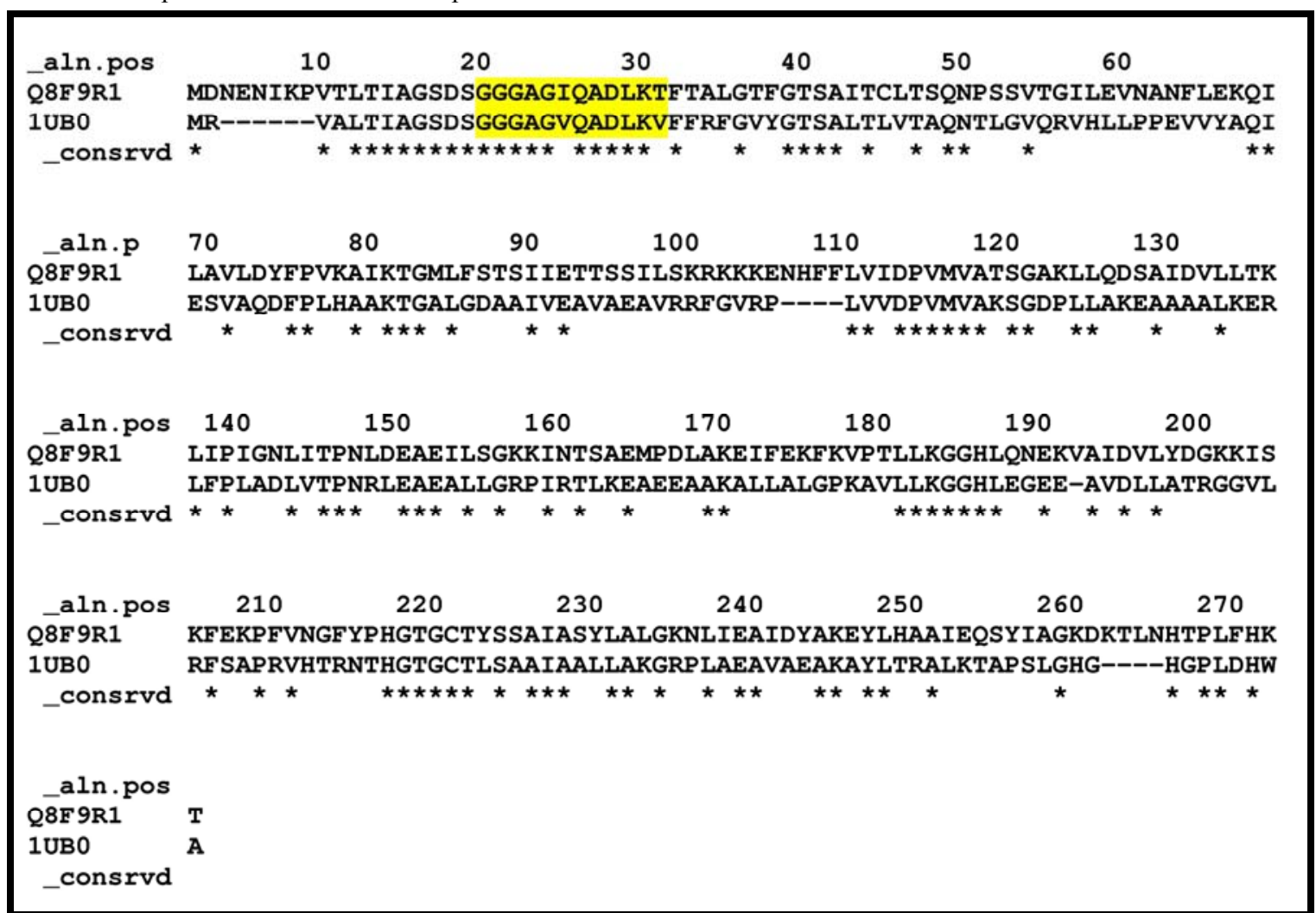

Figure 1: Output of MALIGN script showing the alignment and ATP/GTP binding site (in yellow color)

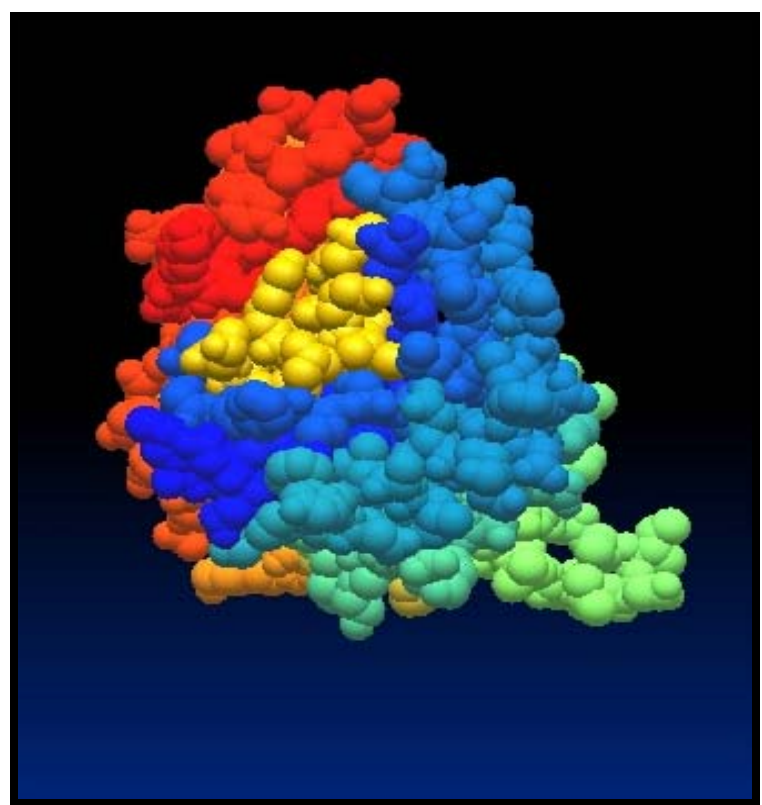

Figure 2: Displaying the ATP/GTP binding site (yellow color) in phosphomethyl pyrimidine kinase enzyme from Leptospira interrogans serovar lai strain 56601 


\section{Bioinformation}

\section{www.bioinformation.net}

Views \& Challenges

\begin{abstract}
The backbone of the obtained model overlapped well with the template structure and the most reliable structure was chosen based on least objective function value and stereo chemical quality of the models using the PROCHECK analysis. [7] In addition, the superimposition was also performed using the SUPERPOSE command in MODELLER and the RMSD (root mean square deviation) between predicted model and template was 0.4 angstrom. The predicted model quality assessment was checked using Ramachandran plot analysis for phi and psi torsion angles. The analysis shows that $90.4 \%$ of residues are found to be in the allowed region of the plot, which is more than the average cut-off of $90 \%$ in most reliable models. [8] The final model has been deposited in PDB and is now publicly accessible [PDB ID: 2G53]. The protein structure predicted is displayed in figure 2 .
\end{abstract}

Results and Discussion:

An active phosphomethyl pyrimidine kinase is a 273 residue long monomer with a molecular mass of $29 \mathrm{kDa}$. A similar result has been documented earlier in E.coli ThiD protein. [10] The protein molecule is an alpha beta structure identified to contain a single large domain (17270 residues). Totally 8 helices and 10 sheets are present in it. All helices and sheets are right hand twisted. All sheets are arranged parallel to each other except for $\beta 4$ and $\beta 9$ sheets, which are anti parallel.

In E.coli, although the phosphorylation reaction of HMP appears to occur by two steps, only one ATP binding motif was found in its ThiD. It is a two step mechanism for phosphorylation is still unclear. [11] In the current work, only a single large domain has been identified. Possibly the phosphorylation process in Leptospira species also follows the not so well understood two step mechanism. Walker et al (1984) suggested that many ATP- and GTP-binding proteins contain a consensus sequence motif [AG]X\%GK[ST]. A sequence similar to this motif, GGGAGIQADLKT, is found in the ThiD sequence starting at position 19 from the $\mathrm{N}$ terminus. Previously a similar sequence motif was also reported in E.coli. [10] This indicates the domain identified in phosphomethyl pyrimidine kinase could be an ATP binding motif.

\section{Conclusion:}

Doxycycline or penicillin drugs are commonly used for treatment of mild or subclinical leptospiral infections. Treatment for severe leptospiral disease is still unclear. Therefore, it is important to design effective antileptospiral drugs. A molecular model of the phosphomethyl pyrimidine kinase from Leptospira interrogans serovar lai strain 56601 is documented in this study. The predicted model is believed to provide valuable insights towards the inhibitor design of Phosphomethyl pyrimidine kinase pertaining to leptospirosis treatment.

\section{References:}

[1] P. N. Levett, Clin. Microbiol. Rev., 14:296 (2001) [PMID: 11292640]

[2] http://bioinfo.hku.hk/Leptolist/

[3] http://www.ebi.ac.uk/

[4] http://www.rcsb.org/pdb/

[5] http://www.ncbi.nlm.nih.gov/blast/

[6] A. Sali \& T. L. Blundell, J. Mol. Biol., 234:779 (1993) [PMID: 8254673]

[7] R. A. Laskowski, et al., J Appl Crystallogr., 26:283 (1993)

[8] G. N. Ramachandran, et al., J. Mol. Biol., 7:95 (1963) [PMID: 13990617]

[9] http://www.genome.ad.jp/kegg/

[10] J. E. Walker, et al., J. Biochim. Biophys. Acta., 768: 164 (1984)

[11] T. Mizote, et al., Microbiology, 145:495 (1999)

Citation: Umashankar et al., Bioinformation by V. S. Mathura

License statement: This is an open-access article, which permits unrestricted use, distribution, and reproduction in any medium, for non-commercial purposes, provided the original author and source are credited. 